\title{
Endothelial to mesenchymal transition: a precursor to post-COVID-19 interstitial pulmonary fibrosis and vascular obliteration?
}

To the Editor:

We read with interest the recent editorial by HuERTAs et al. [1], highlighting the importance of endothelial cell dysfunction in patients with severe acute respiratory syndrome coronavirus 2 (SARS-CoV-2) infection. The authors have made some very fascinating insights, and we would like to take this discussion further, especially emphasising the role of endothelial cells in initiating post-infection pulmonary fibrosis and vascular remodelling. The angiotensin-converting enzyme 2 (ACE2) has been suggested as the primary receptor for mediating SARS-CoV-2 entry into the host cells. Apart from ACE2, the other key players that facilitate SARS-CoV-2 entry, includes transmembrane serine protease 2 (TMPRSS2), furin, sialic acid and the extracellular matrix metalloproteinase inducer (CD147) [1]. In their article, the authors provide compelling comprehensions from a study comparing post mortem lung tissues from patients who died from coronavirus disease 2019 (COVID-19), acute respiratory distress syndrome due to influenza A (H1N1) infection and those from age-matched, uninfected control lungs [1,2]. A crucial inference was the connections between the significant increase in ACE2 positive endothelial cells and the substantial change in endothelial cell morphology, disruption of intercellular junctions, cell swelling, and the breakdown of the underlying basement membrane, all indicative of vascular structural modification in tune to the process of endothelial to mesenchymal transition (EndMT) [3]. Considering the implications for post-COVID-19 pulmonary fibrosis and vascular destruction seen in this infectious pathology, we believe that the role of EndMT in disease manifestation could be consequential.

EndMT occurs when endothelial cells respond to an external insult or an internal pathological condition, transforming themselves into a more aggressive mesenchymal state, causing irreversible vascular damage or fibrosis [3]. The process of EndMT has been considered as one of the major contributors to several other pathological conditions, such as pulmonary arterial hypertension, arterial fibrosis, atherosclerosis, cardiac fibrosis and malignancy [3, 4]. Endothelial cells undergoing EndMT loose endothelial characteristics, such as a change in morphology, loss of vascular endothelial cadherins (VE-cadherins), CD31 and Tie1/2 with subsequent increase in mesenchymal proteins such as $\mathrm{N}$-cadherin, fibroblast specific protein-1 or S100A4, fibronectin, vimentin, SM22- $\alpha$, calponin and $\alpha$-smooth muscle actin [3]. As a part of the process, the basement membrane underlying endothelial cells gets disrupted, facilitating the migration of cells. This occurs through active proteolytic degradation of basement membrane collagen by matrix metalloproteinases mediated by the transitioning cells $[3,5]$.

Сомвет et al. [6], in an interesting case study published in European Respiratory Journal, reported rapid onset of honeycombing fibrosis in a patient with COVID-19, with typical similarity to idiopathic pulmonary fibrosis (IPF); similar questions were raised by SPAGNOLO et al. [7] regrading post-COVID-19 pulmonary fibrosis. In histopathological micrographs from patients with IPF, we observed high ACE2 expression in the intimal, medial and adventitial layers of the pulmonary arteries. Same areas also had high expression of mesenchymal proteins, such as S100A4 and vimentin, indicative of active EndMT. Furthermore, Movat pentachrome stain showed prominent intimal thickening and luminal narrowing (figure 1). It is quite possible that SARS-CoV-2 may lead to such endothelial cell dysfunction.

@ERSpublications

Endothelial to mesenchymal transition (EndMT) could lead to post-COVID-19 pulmonary fibrosis and vascular remodelling https://bit.ly/2QqSKxT

Cite this article as: Eapen MS, Lu W, Gaikwad AV, et al. Endothelial to mesenchymal transition: a precursor to post-COVID-19 interstitial pulmonary fibrosis and vascular obliteration? Eur Respir J 2020; 56: 2003167 [https://doi.org/10.1183/13993003.03167-2020]. 


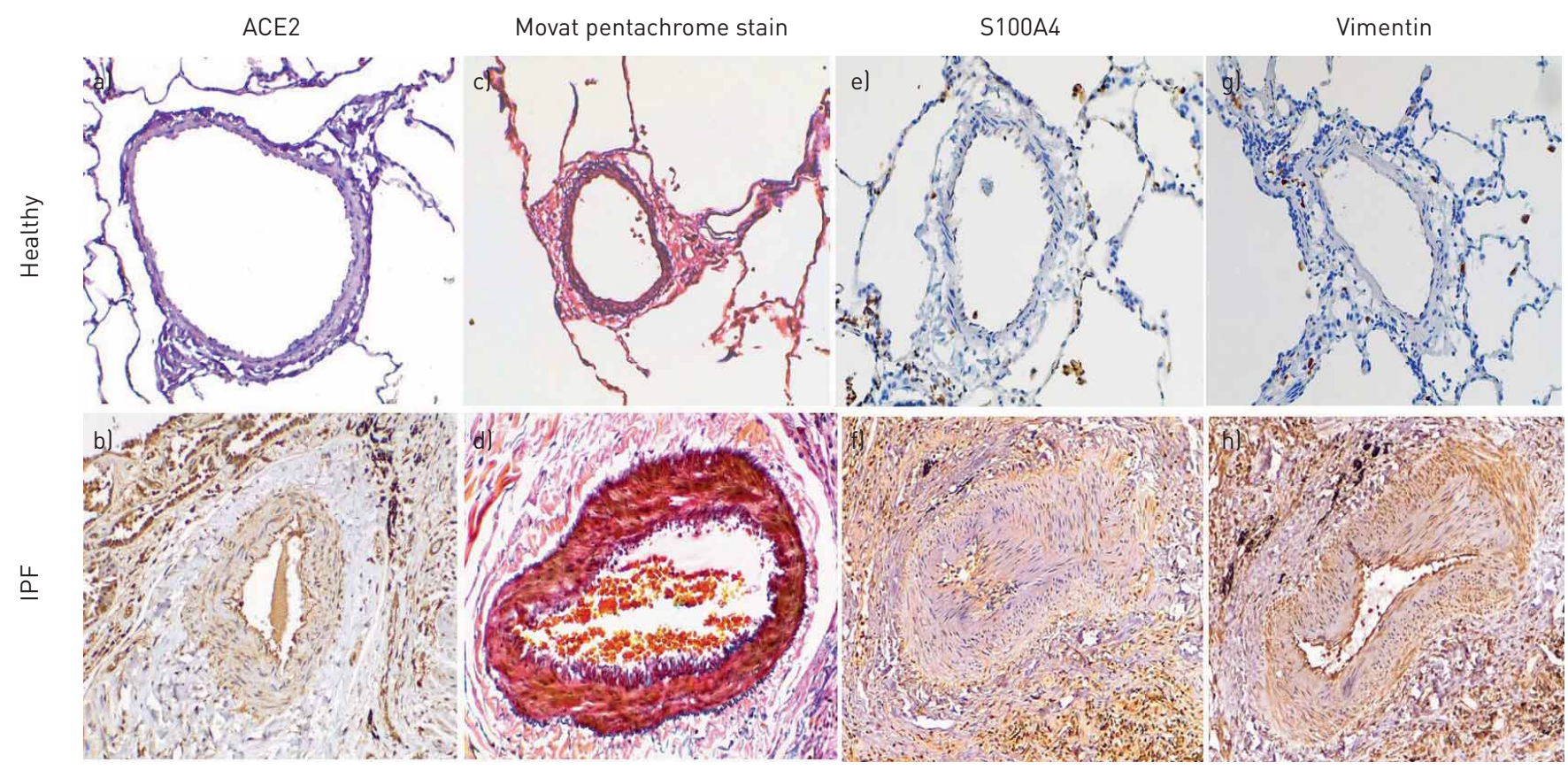

FIGURE 1 Histopathological micrographs of pulmonary arteries from idiopathic pulmonary fibrosis (IPF) patients compared to healthy normal controls (original magnification 20x). a, b) Pulmonary arteries after angiotensin-converting enzyme 2 (ACE2) immunostaining, with high expression in intimal, medial and adventitial layers. c, d) Movat pentachrome staining; note the prominent intimal thickening and luminal narrowing. e, f) Pulmonary arteries after immunohistochemistry with S100A4 and $\mathrm{g}, \mathrm{h}$ ) with vimentin antibodies. The artery wall thickening in IPF patients along with increased expression of mesenchymal biomarkers S100A4 and vimentin are suggestive of active endothelial to mesenchymal transition contributing to vascular remodelling. We propose similar pathology could be driven by severe acute respiratory syndrome coronavirus 2 infection (coronavirus disease 2019) through ACE2 expression in the endothelium as shown in panels a and b. IPF tissue was collected under ethics approval from Alfred Health ethics committee (ethics ID: 336-13) and normal control tissues were provided by the James Hogg Lung Registry, the University of British Columbia (ethics ID: H00-50110).

These observations also pose a concern regarding smokers and patients with COPD. Indeed, this patient population is highly susceptible to SARS-CoV-2 infection, as smoking has been observed to increase ACE2 expression $[8,9]$. It is also essential to note in this context that these patients may be highly compromised to fibrotic conditions post-COVID-19 recovery, as similar endothelial cell dysfunction is quite possible in the vasculature of smokers and patients with COPD, and the underlying mechanism of EndMT might be central to this pathology [5]. The other cell type that could also contribute to fibrosis is type-II pneumocytes. These cells are quite hyperplastic and hyperproliferative in IPF [10], and could further contribute to fibrosis in the long term after patients have recovered from the SARS-CoV-2 infection. Type-II pneumocytes could have similar implications in smokers and patients with COPD.

Like EndMT, epithelial to mesenchymal transition (EMT) is also known to play a crucial role in organ fibrosis and epithelial cell malignancy [5]. We have previously reported that it is an active process in smokers and patients with COPD, both in small and large airways [5]. In this process, epithelial cells transform into a mesenchymal phenotype accompanied by basement membrane degradation, loss of epithelial and gain of mesenchymal proteins [5]. It will be important to see if this insidious infection also leads to enhanced epithelial cell activation through EMT, contributing again to post-COVID-19 pulmonary fibrosis.

Taken together it appears endothelial and epithelial cells may play a prominent role in post-COVID-19 fibrosis. Patients with other disease conditions, such as COPD or IPF, might fare worse after an initial recovery from COVID-19. The process of EndMT and EMT might be central to post-COVID-19 pulmonary fibrosis. There is an urgent need to understand these mechanisms in the context of SARS-CoV-2 manifestations for early interventions. We should be well prepared to deal with the aftermath of COVID-19.

Mathew Suji Eapen $\oplus^{1}$, Wenying Lu$^{1}$, Archana Vijay Gaikwad ${ }^{1}$, Prem Bhattarai ${ }^{1}$, Collin Chia ${ }^{1,2}$, Ashutosh Hardikar ${ }^{1,3}$, Greg Haug ${ }^{1,2}$ and Sukhwinder Singh Sohal ${ }^{1}$

${ }^{1}$ Respiratory Translational Research Group, Department of Laboratory Medicine, School of Health Sciences, College of Health and Medicine, University of Tasmania, Launceston, Australia. ${ }^{2}$ Department of Respiratory Medicine, Launceston General Hospital, Launceston, Australia. ${ }^{3}$ Department of Cardiothoracic Surgery, Royal Hobart Hospital, Hobart, Australia. 
Correspondence: Sukhwinder Singh Sohal, Respiratory Translational Research Group, Department of Laboratory Medicine, School of Health Sciences, College of Health and Medicine, University of Tasmania, Locked Bag - 1322, Newnham Drive, Launceston, Tasmania 7248, Australia. E-mail: sssohal@utas.edu.au

Received: 17 Aug 2020| Accepted: 20 Aug 2020

Acknowledgements: Clifford Craig Foundation Launceston General Hospital, Lung Foundation Australia, The Alfred Hospital Melbourne and James Hogg Lung Registry, The University of British Columbia, Canada.

Conflict of interest: None declared.

\section{References}

1 Huertas A, Montani D, Savale L, et al. Endothelial cell dysfunction: a major player in SARS-CoV-2 infection (COVID-19)? Eur Respir J 2020; 56: 2001634.

2 Ackermann M, Verleden SE, Kuehnel M, et al. Pulmonary vascular endothelialitis, thrombosis, and angiogenesis in Covid-19. N Engl J Med 2020; 383: 120-128.

3 Gaikwad AV, Eapen MS, McAlinden KD, et al. Endothelial to mesenchymal transition (EndMT) and vascular remodeling in pulmonary hypertension and idiopathic pulmonary fibrosis. Expert Rev Respir Med 2020: in press [https://doi.org/10.1080/17476348.2020.1795832].

4 Kovacic JC, Dimmeler S, Harvey RP, et al. Endothelial to mesenchymal transition in cardiovascular disease: JACC state-of-the-art review. J Am Coll Cardiol 2019; 73: 190-209.

5 Sohal SS. Epithelial and endothelial cell plasticity in chronic obstructive pulmonary disease (COPD). Respir Investig 2017; 55: 104-113.

6 Combet M, Pavot A, Savale L, et al. Rapid onset honeycombing fibrosis in spontaneously breathing patient with COVID-19. Eur Respir J 2020; 56: 2001808.

7 Spagnolo P, Balestro E, Aliberti S, et al. Pulmonary fibrosis secondary to COVID-19: a call to arms? Lancet Respir Med 2020; 8: 750-752

8 Brake SJ, Barnsley K, Lu W, et al. Smoking upregulates angiotensin-converting enzyme-2 receptor: a potential adhesion site for novel coronavirus SARS-CoV-2 (Covid-19). J Clin Med 2020; 9: 841.

9 Leung JM, Yang CX, Tam A, et al. ACE-2 expression in the small airway epithelia of smokers and COPD patients: implications for COVID-19. Eur Respir J 2020; 55: 2000688.

10 Selman M, Pardo A. Role of epithelial cells in idiopathic pulmonary fibrosis: from innocent targets to serial killers. Proc Am Thorac Soc 2006; 3: 364-372. 